\title{
Predicting Early and Late Readmissions Following Cytoreductive Surgery and Hyperthermic Intraperitoneal Chemotherapy
}

\author{
Eui Whan Moon, BS, MD ${ }^{1}$, Jolene Si Min Wong, MBBS, MMed, FRCS ${ }^{1}$, Amanda Hui Min See, MBBS, FRCS $^{1}$, \\ Whee Sze Ong, MAppStats ${ }^{2}$, Chee Ann Tan, Medical student ${ }^{1}$, Chin-Ann Johnny Ong, MBBS, MMed, FRCS, \\ PhD $^{1,3,4,5}$, Claramae Shulyn Chia, MBBS, MMed, FRCS ${ }^{1,4}$, Khee Chee Soo, MBBS, MD, FRACS (Australia), \\ FACS $^{1,4}$, Melissa Ching Ching Teo, MBBS, MMed, FRCS, MPH ${ }^{1,4}$, and Grace Hwei Ching Tan, MBBS, MMed, \\ FRCS $^{1}$
}

${ }^{1}$ Division of Surgery and Surgical Oncology, Department of Sarcoma, Peritoneal and Rare Tumours (SPRinT), National Cancer Centre Singapore, Singapore, Singapore; ${ }^{2}$ Department of Clinical Trials and Epidemiological Sciences, National Cancer Centre Singapore, Singapore, Singapore; ${ }^{3}$ Laboratory of Applied Human Genetics, Division of Medical Sciences, National Cancer Centre Singapore, Singapore, Singapore; ${ }^{4}$ SingHealth Duke-NUS Oncology Academic Clinical Program, Duke-NUS Medical School, Singapore, Singapore; ${ }^{5}$ Institute of Molecular and Cell Biology, A*STAR Research Entities, Singapore, Singapore

\begin{abstract}
Background. Postoperative readmissions not only burden the healthcare system but may also affect clinical outcomes of cancer patients. Despite this, little is known about readmissions after cytoreductive surgery (CRS) and hyperthermic intraperitoneal chemotherapy (HIPEC), or their impact on survival outcomes.

Patients and Methods. A single-institution retrospective cohort study of CRS-HIPEC procedures from April 2001 and September 2019 was performed. Early readmission (ERA) was defined as hospitalization within 30 days of discharge post-CRS/HIPEC, while late readmission (LRA) was defined as hospitalization between day 31 and 90 after discharge. Patient demographic, oncological, and perioperative factors were analyzed to identify predictors of readmission, and comparison of survival outcomes was performed.
\end{abstract}

Results. Overall, 342 patients who underwent CRSHIPEC were included in the study. The incidence of ERA and LRA was $18.5 \%$ and $7.4 \%$, respectively. High-grade

(C) The Author(s) 2021

First Received: 30 April 2021

Accepted: 23 June 2021;

Published Online: 24 July 2021

G. H. C. Tan, MBBS, MMed, FRCS

e-mail: grace.tan.h.c@singhealth.com.sg postoperative complication was the only independent predictor of ERA (HR 3.64, 95\% CI 1.47-9.02), while comorbid hypertension (HR 2.71, 95\% CI 1.17-6.28) and stoma creation (HR 2.83, 95\% CI 1.23-6.50) were independent predictors for LRA. Patients with readmission had significantly worse disease-free survival than patients who had no readmission (NRA) (LRA 1.1 years, ERA 1.2 years, NRA 1.8 years, $p=0.002$ ), and patients with LRA had worse median overall survival $(2.1$ years $)$ than ERA patients (3.3 years) or patients without readmission (4.4 years) $(p<0.001)$.

Conclusions. Readmission following CRS-HIPEC is associated with adverse survival outcomes. In particular, LRA may portend worse prognosis than ERA.

Over the past two decades, cytoreductive surgery (CRS) and hyperthermic intraperitoneal chemotherapy (HIPEC) have gradually gained acceptance as a treatment option for selected patients with peritoneal surface-based malignancies secondary to gastrointestinal or gynecological primaries. ${ }^{1-3}$ Complete cytoreduction during CRS is one of the most important predictors of survival outcomes. ${ }^{4}$ Therefore, it is common for multivisceral resection to be performed during CRS to ensure eradication of all macroscopic disease. ${ }^{5}$ As a result, reported rates of postoperative morbidity following CRS-HIPEC range from 10 to $50 \%$, and mortality from 1 to $6 \% .^{6,7}$ 
In addition to morbidity and mortality, another postoperative metric attracting interest in the literature is postoperative readmission (RA) rates. Hospital readmissions after cancer-related surgeries not only contribute to higher costs of care but may also be associated with poorer clinical outcomes. For colon cancer-related colectomies, 1-year mortality for patients with 30-day RA versus without RA was reported to be $16 \%$ versus $7 \%$, respectively, ${ }^{8}$ and similar trends were reported for patients with 90-day RA after surgery for bladder, esophageal, lung, and pancreatic cancers. ${ }^{9}$ Thirty-day RA rates post CRS-HIPEC have been reported to be between 11 and 24\%, indications for which include digestive complications, pain, infection, and venous thromboembolism. ${ }^{10-13}$ Late RA occurring up to postoperative day 90 has been reported to occur at rates of up to $7.8-21 \% .^{14,15}$ Postoperative RA in patients who have undergone CRS-HIPEC not only poses a heavy financial burden on the healthcare system, but may also have significant implications for survival outcomes, as suggested by existing data on RA after other oncological surgeries.

Despite this, there is a paucity of data on predictors for hospital RA after CRS-HIPEC among the Asian population, and even fewer studies that evaluate its association with survival rates. To address these knowledge gaps, the aims of this study are to identify risk factors associated with early and late RA post CRS-HIPEC and their impact on oncologic outcomes.

\section{PATIENTS AND METHODS}

Ethical approval from the SingHealth Centralised Institutional Review Board was obtained for the conduct of this retrospective cohort study. Data were retrieved from a prospectively maintained database of patients who had undergone CRS-HIPEC at National Cancer Centre Singapore.

\section{Patient Selection}

Patients were selected for CRS-HIPEC upon review and recommendation by a multidisciplinary tumor board discussion. All patients selected had Eastern Cooperative Group (ECOG) performance status of either 0 or 1 and no distant metastases as verified by either computed tomography (CT) scan or positron emission tomography (PET)CT scan.

Patients who underwent CRS-HIPEC at our institution between April 2001 and September 2019 and were discharge from hospital were included in the study. Repeat CRS-HIPEC procedures of patients during the study period were excluded.

\section{CRS-HIPEC}

We previously described how CRS-HIPEC was performed at our institution. ${ }^{16}$ In brief, cytoreduction was performed as described by Sugarbaker. ${ }^{17}$ An intraperitoneal chemotherapy agent appropriate for the patient's malignancy type was prescribed by the medical oncologist and administered intraoperatively via a hyperthermia pump into a closed abdomen at $41-42{ }^{\circ} \mathrm{C}$ for $60 \mathrm{~min}$. The Peritoneal Cancer Index $(\mathrm{PCI})^{17}$ was used to document the extent of peritoneal disease, while the completeness of cytoreduction (CC) score $^{18}$ was recorded to quantify the extent of cytoreduction.

\section{Postoperative Care}

Following CRS-HIPEC, patients were typically monitored in the surgical intensive care unit (SICU) or highdependency unit as deemed necessary by the primary surgeon and anesthetist. Postoperative complications were documented according to the Clavien-Dindo classification. ${ }^{19}$ Upon discharge, outpatient follow-up appointments were given at 1 week postdischarge, followed by a 1-month appointment, and thereafter 3-monthly appointments for 1 year, and 6-monthly appointments thereafter. Adjuvant chemotherapy was offered by medical oncologist as appropriate, and recurrences were documented.

\section{Key Definitions}

Patients were categorized into three readmission categories:

(1) Early readmission (ERA) was defined as the first unplanned (i.e., emergency, nonelective) hospitalization within 30 days (inclusive) post discharge from index CRS/HIPEC.

(2) Late readmission (LRA) was defined as hospitalization occurring from 31 to 90 days after discharge from index CRS/HIPEC.

(3) No readmission (NRA) was defined as no readmission within 90 days after discharge from index CRSHIPEC.

Disease-free survival (DFS) was defined as duration between CRS-HIPEC and first recurrence or death from any cause, whichever occurred first, while overall survival (OS) was defined as duration between CRS-HIPEC and death from any cause.

Patients who did not experience the stated events for DFS and OS were censored at their last follow-up date. 


\section{Statistics}

For analysis of time to ERA and time to LRA, NRA patients were censored at day 90 post discharge from index CRS-HIPEC. For analysis of time to ERA, LRA and NRA patients were censored at day 30 post discharge from index CRS-HIPEC.

Patient demographic, oncological, operative, and postoperative factors were compared between ERA, LRA, and NRA using Fisher's exact test and Kruskal-Wallis test for categorical and continuous variables, respectively. Cumulative incidence rate of RA was derived based on one minus the Kaplan-Meier estimate of the survival function for time to RA. Univariate and multivariable Cox proportional hazard $(\mathrm{PH})$ regression models were used to examine the association of various factors with time to ERA and time to LRA. Variables with univariate $p<0.05$ were included in the multivariable model. PH assumption was verified based on Schoenfeld residuals.

Follow-up duration was measured from discharge from CRS-HIPEC until date of last follow-up and estimated using the inverse Kaplan-Meier method. DFS and OS were estimated using Kaplan-Meier method. Differences in DFS and OS between patients in the three RA groups were compared using log-rank test.

Two-sided $p$ value $<0.05$ was considered statistically significant. All analyses were performed using SAS version 9.4 (SAS Institute Inc., Cary, NC).

\section{RESULTS}

Overall, 342 patients underwent CRS-HIPEC during the study duration. The demographics of the patients included in the study are summarized in Table 1. Our patient population had median age of 55 years, with ECOG status of 0 $(87 \%)$ or $1(12 \%)$. Colorectal cancer was the most common primary, accounting for nearly $40 \%$ of the cohort, followed by appendiceal cancer $(25 \%)$, ovarian cancer $(21 \%)$, and primary peritoneal disease (5\%). Median PCI was 9, and after a median operative time of $495 \mathrm{~min}$ with median estimated blood loss of $1000 \mathrm{~mL}, \mathrm{CC}-0$ was achieved in $82 \%$ of cases, while CC-1 was achieved in $11 \%$. Median length of hospitalization for CRS-HIPEC was 11 days (Table 2).

Sixty patients had ERA, 23 had LRA, and 259 had NRA within 90 days post discharge from CRS-HIPEC. Median time to ERA and LRA was 8 and 51 days, respectively.

Median age between RA groups was comparable, as was the distribution of ECOG status. Ovarian (ERA 28\% versus LRA 17\%), appendiceal (ERA 20\% versus LRA 9\%), and peritoneal (ERA $10 \%$ versus LRA $4.3 \%$ ) primaries were more common in ERA group, while colorectal primary was more common is LRA group (LRA $48 \%$ versus ERA 32\%), but these differences in distribution did not reach statistical significance. Median PCI score was comparable between RA groups, and CC-0 score was achieved in $77 \%$ of LRA group and $91 \%$ of ERA group $(p=0.155)$. The most common cytoreductive procedure performed across both readmission groups was subdiaphragmatic stripping, followed by colectomy and small bowel resection.

\section{Causes of Readmission Following CRS-HIPEC}

The majority (46\%) of RA were due to gastrointestinal complaints, such as abdominal pain, bloatedness, nausea, and vomiting (Table 3). A total of $15 \%$ of RA were a result of stoma-related complications (e.g., high stoma output), and $8 \%$ from superficial wound infections. Although there was a higher percentage of LRA patients (26\%) with stoma-related readmission compared with ERA patients $(10 \%)$, on balance reasons for RA were similar between these two groups $(p=0.450)$.

\section{Factors Affecting Readmissions Following CRS-HIPEC}

Comparison of operative and postoperative variables showed that, compared with the NRA and LRA groups, patients with ERA had the longest duration of CRS-HIPEC [median $475 \mathrm{~min}$ (NRA) versus $500 \mathrm{~min}$ (LRA) versus 585 min (ERA); $p=0.016]$, highest percentage with grade IIIIV postoperative complication [12\% (NRA) versus $22 \%$ (LRA) versus 35\% (ERA); $p<0.001]$ and longest index hospital admission for CRS-HIPEC [median 11 days (NRA) versus 13 days (LRA) versus 14 days (ERA); $p=$ 0.001] (Tables 1 and 2).

There were significantly more patients who had stoma created at index CRS-HIPEC among LRA group (48\%) than ERA $(40 \%)$ or NRA $(27 \%)(p=0.020)$. Demographic and oncological variables showed no significant difference between the three readmission groups (Table 1).

Univariate Cox regression analysis identified eight significant predictors for ERA: PCI score, intraoperative bladder resection, intraoperative chest tube placement, duration of CRS-HIPEC, CC score, intraoperative blood transfusion, duration of hospital stay, and high-grade postoperative complication (grade III-IV). On multivariate analysis, only high-grade postoperative complication continued to be significant (HR 3.64, 95\% CI 1.47-9.02; relative to no complications) (Table 4). For LRA, the variables significant on univariate analysis, which also remained significant on multivariate analysis, were presence of hypertension (HR 2.71, 95\% CI 1.17-6.28) and stoma creation (HR 2.83, 95\% CI 1.23-6.50) (Table 5). 
TABLE 1. Demographics, clinical, and treatment characteristics

\begin{tabular}{|c|c|c|c|c|c|}
\hline & Total $(N=342)$ & NRA $(n=259)$ & ERA $(n=60)$ & LRA $(n=23)$ & $p$ value \\
\hline \multicolumn{6}{|l|}{ Demographic } \\
\hline Age at CRS-HIPEC (years) & $55(14-79)$ & $54(22-79)$ & $56(25-76)$ & $57(14-74)$ & 0.677 \\
\hline \multicolumn{6}{|l|}{ Gender } \\
\hline Female & $237(69.3)$ & $179(69.1)$ & $44(73.3)$ & $14(60.9)$ & \multirow[t]{2}{*}{0.537} \\
\hline Male & $105(30.7)$ & $80(30.9)$ & $16(26.7)$ & $9(39.1)$ & \\
\hline \multicolumn{6}{|l|}{ Ethnicity } \\
\hline Chinese & $256(74.9)$ & $187(72.2)$ & $49(81.7)$ & $20(87.0)$ & \multirow[t]{4}{*}{0.482} \\
\hline Malay & $23(6.7)$ & $17(6.6)$ & $5(8.3)$ & $1(4.3)$ & \\
\hline Indian & $16(4.7)$ & $15(5.8)$ & $1(1.7)$ & $0(-)$ & \\
\hline Other & $47(13.7)$ & $40(15.4)$ & $5(8.3)$ & $2(8.7)$ & \\
\hline \multicolumn{6}{|l|}{ Clinical } \\
\hline \multicolumn{6}{|l|}{ ECOG performance status } \\
\hline 0 & $296(86.5)$ & $224(86.5)$ & $53(88.3)$ & $19(82.6)$ & \multirow[t]{3}{*}{0.782} \\
\hline 1 & $41(12.0)$ & $30(11.6)$ & 7 (11.7) & $4(17.4)$ & \\
\hline Missing & $5(1.5)$ & $5(1.9)$ & $0(-)$ & $0(-)$ & \\
\hline \multicolumn{6}{|l|}{ Comorbidities } \\
\hline Absent & $124(36.3)$ & $97(37.5)$ & $20(33.3)$ & $7(30.4)$ & \multirow[t]{2}{*}{0.766} \\
\hline Present & $218(63.7)$ & $162(62.5)$ & $40(66.7)$ & $16(69.6)$ & \\
\hline \multicolumn{6}{|l|}{ Type of comorbidities } \\
\hline Hypertension & $95(27.8)$ & $64(24.7)$ & $21(35.0)$ & $10(43.5)$ & 0.056 \\
\hline Diabetes & $42(12.3)$ & $30(11.6)$ & $10(16.7)$ & $2(8.7)$ & 0.514 \\
\hline Hyperlipidemia & $68(19.9)$ & $48(18.5)$ & $16(26.7)$ & $4(17.4)$ & 0.341 \\
\hline Ischemic heart disease & $9(2.6)$ & $7(2.7)$ & $2(3.3)$ & $0(-)$ & 0.827 \\
\hline COPD & $2(0.6)$ & $2(0.8)$ & $0(-)$ & $0(-)$ & 1.000 \\
\hline Asthma & $7(2.0)$ & $3(1.2)$ & $3(5.0)$ & $1(4.3)$ & 0.065 \\
\hline Other malignancy & $23(6.7)$ & $16(6.2)$ & $5(8.3)$ & $2(8.7)$ & 0.668 \\
\hline Others & $132(38.6)$ & $105(40.5)$ & $21(35.0)$ & $6(26.1)$ & 0.334 \\
\hline \multicolumn{6}{|l|}{ Primary tumor site } \\
\hline Colorectal $^{\mathrm{a}}$ & $129(37.7)$ & $99(38.2)$ & $19(31.7)$ & $11(47.8)$ & \multirow[t]{6}{*}{0.052} \\
\hline Ovarian $^{\mathrm{b}}$ & $73(21.3)$ & $52(20.1)$ & $17(28.3)$ & $4(17.4)$ & \\
\hline Peritoneal & $18(5.3)$ & $11(4.2)$ & $6(10.0)$ & $1(4.3)$ & \\
\hline Appendix & $87(25.4)$ & $73(28.2)$ & $12(20.0)$ & $2(8.7)$ & \\
\hline Mesothelioma & $13(3.8)$ & $7(2.7)$ & $4(6.7)$ & $2(8.7)$ & \\
\hline Others & $22(6.4)$ & $17(6.6)$ & $2(3.3)$ & $3(13.0)$ & \\
\hline PCI score & $9(0-39)$ & $8(0-39)$ & $13(0-36)$ & $14(0-31)$ & 0.088 \\
\hline No. of patients with nonmissing data & 310 & 235 & 54 & 21 & \\
\hline \multicolumn{6}{|l|}{ Ascites } \\
\hline Absent & $200(58.5)$ & $161(62.2)$ & $29(48.3)$ & $10(43.5)$ & \multirow[t]{3}{*}{0.113} \\
\hline Present & $94(27.5)$ & $63(24.3)$ & $21(35.0)$ & $10(43.5)$ & \\
\hline Missing & $48(14.0)$ & $35(13.5)$ & $10(16.7)$ & $3(13.0)$ & \\
\hline \multicolumn{6}{|l|}{ Treatment } \\
\hline \multicolumn{6}{|l|}{ CRS procedure } \\
\hline Subdiaphragmatic stripping & $125(36.5)$ & $88(34.0)$ & $27(45.0)$ & $10(43.5)$ & 0.201 \\
\hline Gastrectomy & $19(5.6)$ & $15(5.8)$ & $4(6.7)$ & $0(-)$ & 0.626 \\
\hline Colectomy & $110(32.2)$ & $79(30.5)$ & $24(40.0)$ & $7(30.4)$ & 0.370 \\
\hline Small bowel resection & $62(18.1)$ & $42(16.2)$ & $15(25.0)$ & $5(21.7)$ & 0.232 \\
\hline Splenectomy & $58(17.0)$ & $42(16.2)$ & $14(23.3)$ & $2(8.7)$ & 0.274 \\
\hline THBSO & $49(14.3)$ & $33(12.7)$ & $11(18.3)$ & $5(21.7)$ & 0.268 \\
\hline
\end{tabular}


TABLE 1. continued

\begin{tabular}{|c|c|c|c|c|c|}
\hline & Total $(N=342)$ & NRA $(n=259)$ & ERA $(n=60)$ & LRA $(n=23)$ & $p$ value \\
\hline Cholecystectomy & $57(16.7)$ & $44(17.0)$ & $11(18.3)$ & $2(8.7)$ & 0.618 \\
\hline Bladder resection & $9(2.6)$ & $5(1.9)$ & $4(6.7)$ & $0(-)$ & 0.131 \\
\hline Other procedure(s) & $100(29.2)$ & $77(29.7)$ & $15(25.0)$ & $8(34.8)$ & 0.623 \\
\hline \multicolumn{6}{|l|}{ HIPEC agent } \\
\hline Cisplatin & 109 (31.9) & $74(28.6)$ & $25(41.7)$ & $10(43.5)$ & \multirow[t]{4}{*}{0.090} \\
\hline Mitomycin C & $215(62.9)$ & $172(66.4)$ & $32(53.3)$ & $11(47.8)$ & \\
\hline Others $^{c}$ & $12(3.5)$ & $7(2.7)$ & $3(5.0)$ & $2(8.7)$ & \\
\hline Missing & $6(1.8)$ & $6(2.3)$ & $0(-)$ & $0(-)$ & \\
\hline Duration of CRS-HIPEC, mins & $495(245-1070)$ & $475(245-1070)$ & $585(285-1020)$ & $500(310-795)$ & 0.016 \\
\hline No. of patients with nonmissing data: & 297 & 221 & 54 & 22 & \\
\hline \multicolumn{6}{|l|}{ CC score } \\
\hline 0 & 279 (81.6) & $212(81.9)$ & $46(76.7)$ & $21(91.3)$ & \multirow[t]{5}{*}{0.155} \\
\hline 1 & $36(10.5)$ & $26(10.0)$ & $10(16.7)$ & $0(-)$ & \\
\hline 2 & $4(1.2)$ & $3(1.2)$ & $1(1.7)$ & $0(-)$ & \\
\hline 3 & $1(0.3)$ & $0(-)$ & $1(1.7)$ & $0(-)$ & \\
\hline Missing & $22(6.4)$ & $18(6.9)$ & $2(3.3)$ & $2(8.7)$ & \\
\hline \multicolumn{6}{|l|}{ Chest tube placement } \\
\hline No & $154(45.0)$ & $126(48.6)$ & $20(33.3)$ & $8(34.8)$ & \multirow[t]{3}{*}{0.096} \\
\hline Yes & $175(51.2)$ & $122(47.1)$ & $39(65.0)$ & $14(60.9)$ & \\
\hline Missing & $13(3.8)$ & $11(4.2)$ & $1(1.7)$ & $1(4.3)$ & \\
\hline \multicolumn{6}{|l|}{ Stoma creation } \\
\hline No & $238(69.6)$ & $190(73.4)$ & $36(60.0)$ & $12(52.2)$ & \multirow[t]{2}{*}{0.020} \\
\hline Yes & $104(30.4)$ & $69(26.6)$ & $24(40.0)$ & $11(47.8)$ & \\
\hline Estimated blood loss (ml) & $1000(0-11,000)$ & $900(0-11,000)$ & $1000(200-5100)$ & $800(0-3500)$ & 0.439 \\
\hline No. of patients with nonmissing data: & 327 & 248 & 58 & 21 & \\
\hline \multicolumn{6}{|l|}{ Intraoperative blood transfusions } \\
\hline No & $110(32.2)$ & $90(34.7)$ & $13(21.7)$ & $7(30.4)$ & \multirow[t]{3}{*}{0.236} \\
\hline Yes & $224(65.5)$ & $163(62.9)$ & $46(76.7)$ & $15(65.2)$ & \\
\hline Missing & $8(2.3)$ & $6(2.3)$ & $1(1.7)$ & $1(4.3)$ & \\
\hline
\end{tabular}

$N R A$ No readmission, ERA Early readmission, LRA Late readmission, CRS Cytoreduction surgery, HIPEC Hyperthermic intraperitoneal chemotherapy, ECOG Eastern Cooperative Oncology Group, COPD Chronic obstructive pulmonary disease, PCI Peritoneal cancer index, THBSO Total abdominal hysterectomy with bilateral salpingo-oophorectomy, CC Completeness of cytoreduction

Data presented as median (range) if variable is continuous, and number (\%) if variable is categorical

$p$ value based on Kruskal-Wallis test for continuous variable and Fisher's exact test for categorical variable

${ }^{a}$ Included one patient who had an additional primary tumor in endometrium

${ }^{\mathrm{b}}$ Included one patient who had an additional primary gastric tumor

${ }^{\mathrm{c}}$ Included doxorubicin, oxaliplatin, and fluorouracil

\section{Relationship Between Readmission and Survival Outcomes}

The median follow-up of NRA, ERA, and LRA groups was $2.0,4.5$, and 1.9 years, respectively.

\section{Disease-Free Survival}

Patients with readmission had significantly worse DFS than NRA patients (Fig. 1). Median DFS was 1.1 years (95\% CI $0.4-1.8$ years) for LRA patients and 1.2 years
(95\% CI 0.6-1.8 years) for ERA patients, both being lower than the corresponding 1.8 years (95\% CI $1.4-2.2$ years) for NRA patients $(p=0.002$; Table 6, Fig. 1a).

\section{Overall Survival}

LRA patients had worst median OS (2.1 years, $95 \%$ CI 1.0-3.6 years), followed by ERA patients (3.3 years, $95 \%$ CI 1.8-5.9 years) and NRA patients (4.4 years, 95\% CI $3.5-6.0$ years) $(p<0.001$; Table 6; Fig. $1 b)$. 
TABLE 2. Postoperative characteristics and recurrence

\begin{tabular}{|c|c|c|c|c|c|}
\hline & Total $(N=342)$ & NRA $(n=259)$ & ERA $(n=60)$ & LRA $(n=23)$ & $p$ value \\
\hline \multicolumn{6}{|l|}{ Postoperative complications } \\
\hline No & $145(42.4)$ & $122(47.1)$ & $15(25.0)$ & $8(34.8)$ & \multirow[t]{2}{*}{0.006} \\
\hline Yes & $197(57.6)$ & $137(52.9)$ & $45(75.0)$ & $15(65.2)$ & \\
\hline \multicolumn{6}{|c|}{ Worst grade of postoperative complications } \\
\hline No complication & $145(42.4)$ & $122(47.1)$ & $15(25.0)$ & $8(34.8)$ & \multirow[t]{5}{*}{$<0.001$} \\
\hline G1 & $48(14.0)$ & $37(14.3)$ & $7(11.7)$ & $4(17.4)$ & \\
\hline $\mathrm{G} 2$ & $92(26.9)$ & $69(26.6)$ & $17(28.3)$ & $6(26.1)$ & \\
\hline G3 & $44(12.9)$ & $20(7.7)$ & $19(31.7)$ & $5(21.7)$ & \\
\hline G4 & $13(3.8)$ & $11(4.2)$ & $2(3.3)$ & $0(-)$ & \\
\hline Length of SICU stay (days) & $0(0-40)$ & $0(0-40)$ & $1(0-5)$ & $1(0-3)$ & 0.015 \\
\hline No. of patients with nonmissing data & 341 & 258 & 60 & 23 & \\
\hline Length of hospital stay (days) & $11(5-141)$ & $11(5-141)$ & $14(7-66)$ & $13(8-86)$ & 0.001 \\
\hline No. of recurred patients & 163 & 116 & 32 & 15 & - \\
\hline \multicolumn{6}{|l|}{ Site of relapse among recurred patients: } \\
\hline Peritoneum & $110(67.5)$ & $82(70.7)$ & $18(56.3)$ & $10(66.7)$ & 0.293 \\
\hline Lymph nodes & $36(22.1)$ & $22(19.0)$ & $11(34.4)$ & $3(20.0)$ & 0.181 \\
\hline Lung & $38(23.3)$ & $29(25.0)$ & $5(15.6)$ & $4(26.7)$ & 0.490 \\
\hline Liver & $36(22.1)$ & $22(19.0)$ & $9(28.1)$ & $5(33.3)$ & 0.249 \\
\hline Bone & $7(4.3)$ & $4(3.4)$ & $0(-)$ & $3(20.0)$ & 0.016 \\
\hline Skin & $0(-)$ & $0(-)$ & $0(-)$ & $0(-)$ & - \\
\hline Others & $40(24.5)$ & $28(24.1)$ & 5 (15.6) & 7 (46.7) & 0.083 \\
\hline
\end{tabular}

NRA No readmission, ERA Early readmission, LRA Late readmission, SICU Surgical intensive care unit

Data presented as median (range) if variable is continuous, and number (\%) if variable is categorical

$p$ value based on Kruskal-Wallis test for continuous variable and Fisher's exact test for categorical variable

TABLE 3. Readmission characteristics

\begin{tabular}{|c|c|c|c|c|}
\hline & Total $(N=83)$ & ERA $(n=60)$ & LRA $(n=23)$ & $p$ value \\
\hline \multicolumn{5}{|l|}{ Readmission reason } \\
\hline GI symptoms & $38(45.8)$ & $29(48.3)$ & $9(39.1)$ & \multirow[t]{5}{*}{0.450} \\
\hline Wound infection & $7(8.4)$ & $5(8.3)$ & $2(8.7)$ & \\
\hline Other infection & $4(4.8)$ & $3(5.0)$ & $1(4.3)$ & \\
\hline Stoma related & $12(14.5)$ & $6(10.0)$ & $6(26.1)$ & \\
\hline Others & $22(26.5)$ & $17(28.3)$ & $5(21.7)$ & \\
\hline \multicolumn{5}{|l|}{ Death within 30 days of readmission } \\
\hline Excluding alive patients with $<30$ days follow-up: & 82 & 60 & 22 & \multirow{3}{*}{1.000} \\
\hline No & $81(98.8)$ & $59(98.3)$ & $22(100)$ & \\
\hline Yes & $1(1.2)$ & $1(1.7)$ & $0(-)$ & \\
\hline
\end{tabular}

ERA Early readmission, LRA Late readmission

Data presented as number (\%)

$p$ value based on Fisher's exact test 
TABLE 4. Predictors of early readmission

\begin{tabular}{|c|c|c|c|c|}
\hline & \multicolumn{2}{|l|}{ Univariate Cox } & \multicolumn{2}{|l|}{ Multivariable Cox } \\
\hline & $\mathrm{HR}(95 \% \mathrm{CI})$ & $p$ value & $\mathrm{HR}(95 \% \mathrm{CI})$ & $p$ value \\
\hline Age at CRS-HIPEC (per year increase) & $1.01(0.98-1.03)$ & 0.612 & & \\
\hline Gender: male versus female & $0.79(0.45-1.40)$ & 0.424 & & \\
\hline Ethnicity: Malay versus Chinese & $1.32(0.53-3.31)$ & 0.554 & & \\
\hline Ethnicity: Indian versus Chinese & $0.39(0.05-2.81)$ & 0.350 & & \\
\hline Ethnicity: others versus Chinese & $0.54(0.21-1.35)$ & 0.184 & & \\
\hline ECOG performance status: 1 versus 0 & $1.00(0.46-2.20)$ & 0.998 & & \\
\hline Comorbidities: absent versus present & $0.83(0.48-1.42)$ & 0.489 & & \\
\hline Hypertension: yes versus no & $1.48(0.87-2.52)$ & 0.148 & & \\
\hline Diabetes: yes versus no & $1.61(0.82-3.17)$ & 0.171 & & \\
\hline Hyperlipidemia: yes versus no & $1.47(0.83-2.61)$ & 0.186 & & \\
\hline Ischemic heart disease: yes versus no & $1.29(0.31-5.27)$ & 0.726 & & \\
\hline COPD: yes versus no & UD & 0.985 & & \\
\hline Asthma: yes versus no & $3.07(0.96-9.81)$ & 0.058 & & \\
\hline Other malignancy: yes versus no & $1.42(0.57-3.54)$ & 0.456 & & \\
\hline Other comorbidities: yes versus no & $0.86(0.50-1.45)$ & 0.563 & & \\
\hline Tumor site: ovarian versus colorectal & $1.67(0.87-3.21)$ & 0.125 & & \\
\hline Tumor site: peritoneal versus colorectal & $2.38(0.95-5.95)$ & 0.065 & & \\
\hline Tumor site: appendix versus colorectal & $0.98(0.48-2.02)$ & 0.952 & & \\
\hline Tumor site: mesothelioma versus colorectal & $2.44(0.83-7.19)$ & 0.105 & & \\
\hline Tumor site: others versus colorectal & $0.58(0.14-2.49)$ & 0.464 & & \\
\hline PCI score (per unit increase) & $1.03(1.00-1.06)$ & 0.038 & $0.98(0.94-1.03)$ & 0.446 \\
\hline Had ascites: yes versus no & $1.64(0.93-2.87)$ & 0.086 & & \\
\hline Subdiaphragmatic stripping: yes versus no & $1.49(0.89-2.47)$ & 0.128 & & \\
\hline Gastrectomy: yes versus no & $1.21(0.44-3.34)$ & 0.712 & & \\
\hline Colectomy: yes versus no & $1.52(0.91-2.55)^{\mathrm{a}}$ & 0.111 & & \\
\hline Small bowel resection: yes versus no & $1.56(0.87-2.80)$ & 0.136 & & \\
\hline Splenectomy: yes versus no & $1.53(0.84-2.78)$ & 0.166 & & \\
\hline THBSO: yes versus no & $1.47(0.77-2.83)$ & 0.247 & & \\
\hline Cholecystectomy: yes versus no & $1.17(0.61-2.25)$ & 0.638 & & \\
\hline Bladder resection: yes versus no & $2.99(1.08-8.24)$ & 0.035 & Note $^{\mathrm{b}}$ & \\
\hline Other CRS procedure(s): yes versus no & $0.77(0.43-1.39)$ & 0.389 & & \\
\hline HIPEC agent: mitomycin $\mathrm{C}$ versus cisplatin & $0.64(0.38-1.08)$ & 0.093 & & \\
\hline HIPEC agent: others versus cisplatin & $1.14(0.34-3.77)$ & 0.832 & & \\
\hline Duration of CRS-HIPEC (per 10min increase) & $1.02(1.01-1.04)$ & 0.007 & $1.01(0.98-1.04)$ & 0.528 \\
\hline CC score: $\geq 1$ versus 0 & $1.99(1.06-3.76)$ & 0.034 & $1.49(0.59-3.80)$ & 0.401 \\
\hline Chest tube placement: yes versus no & $1.88(1.10-3.22)$ & 0.022 & $1.12(0.53-2.38)$ & 0.767 \\
\hline Stoma creation: yes versus no & $1.65(0.99-2.77)$ & 0.056 & & \\
\hline Blood loss (per 100-ml increase) & $1.01(0.99-1.03)$ & 0.425 & & \\
\hline Intraoperative blood transfusion: yes versus no & $1.86(1.00-3.44)$ & 0.049 & $1.36(0.61-3.06)$ & 0.450 \\
\hline Postoperative complication: G1-G2 versus none & $1.72(0.90-3.29)$ & 0.098 & $1.86(0.89-3.89)$ & 0.098 \\
\hline Postoperative complication: G3-G4 versus none & $4.49(2.31-8.71)$ & $<0.001$ & $3.64(1.47-9.02)$ & 0.005 \\
\hline Length of SICU stay (per day increase) & $1.00(0.94-1.08)$ & 0.917 & & \\
\hline Length of hospital stay (per day increase) & $1.01(1.00-1.03)$ & 0.028 & $1.00(0.97-1.02)$ & 0.758 \\
\hline
\end{tabular}

HR Hazard ratio, CI Confidence interval, CRS Cytoreduction surgery, HIPEC Hyperthermic intraperitoneal chemotherapy, ECOG Eastern Cooperative Oncology Group, COPD Chronic obstructive pulmonary disease, PCI Peritoneal cancer index, THBSO Total abdominal hysterectomy with bilateral salpingo-oophorectomy, $C C$ Completeness of cytoreduction, SICU Surgical intensive care unit, UD No event in one of the categories

$p$ value based on Wald's test

${ }^{\mathrm{a}}$ Violated PH assumption

${ }^{\mathrm{b}}$ Excluded from model due to small sample size 
TABLE 5. Predictors of late readmission

\begin{tabular}{|c|c|c|c|c|}
\hline & \multicolumn{2}{|l|}{ Univariate Cox } & \multicolumn{2}{|l|}{ Multivariable Cox } \\
\hline & HR $(95 \% \mathrm{CI})$ & $p$ value & HR $(95 \% \mathrm{CI})$ & $p$ value \\
\hline Age at CRS-HIPEC (per year increase) & $1.00(0.97-1.04)$ & 0.888 & & \\
\hline Gender: male versus female & $1.34(0.58-3.08)$ & 0.499 & & \\
\hline Ethnicity: Malay versus Chinese & $0.66(0.09-4.92)$ & 0.686 & & \\
\hline Ethnicity: Indian versus Chinese & UD & 0.990 & & \\
\hline Ethnicity: others versus Chinese & $0.46(0.11-1.98)$ & 0.299 & & \\
\hline ECOG performance status: 1 versus 0 & $1.85(0.63-5.44)$ & 0.263 & & \\
\hline Comorbidities: absent versus present & $0.69(0.28-1.68)$ & 0.415 & & \\
\hline Hypertension: yes versus no & $2.28(1.00-5.21)$ & 0.050 & $2.71(1.17-6.28)$ & 0.020 \\
\hline Diabetes: yes versus no & $0.81(0.19-3.47)$ & 0.779 & & \\
\hline Hyperlipidemia: yes versus no & $0.90(0.31-2.64)$ & 0.843 & & \\
\hline Ischemic heart disease: yes versus no & UD & 0.992 & & \\
\hline COPD: yes versus no & UD & 0.990 & & \\
\hline Asthma: yes versus no & $4.60(0.62-34.12)$ & 0.136 & & \\
\hline Other malignancy: yes versus no & $1.46(0.34-6.22)$ & 0.610 & & \\
\hline Other comorbidities: yes versus no & $0.53(0.21-1.36)$ & 0.187 & & \\
\hline Tumor site: ovarian versus colorectal & $0.76(0.24-2.39)$ & 0.638 & & \\
\hline Tumor site: peritoneal versus colorectal & $0.81(0.11-6.29)$ & 0.841 & & \\
\hline Tumor site: appendix versus colorectal & $0.26(0.06-1.17)$ & 0.079 & & \\
\hline Tumor site: mesothelioma versus colorectal & $2.24(0.50-10.10)$ & 0.295 & & \\
\hline Tumor site: others versus colorectal & $1.43(0.40-5.11)$ & 0.587 & & \\
\hline PCI score (per unit increase) & $1.01(0.97-1.06)$ & 0.646 & & \\
\hline Had ascites: yes versus no & $2.36(0.98-5.66)^{\mathrm{a}}$ & 0.055 & & \\
\hline Subdiaphragmatic stripping: yes versus no & $1.41(0.62-3.21)$ & 0.419 & & \\
\hline Gastrectomy: yes versus no & UD & 0.988 & & \\
\hline Colectomy: yes versus no & $0.99(0.41-2.41)$ & 0.988 & & \\
\hline Small bowel resection: yes versus no & $1.38(0.51-3.71)$ & 0.528 & & \\
\hline Splenectomy: yes versus no & $0.45(0.11-1.91)$ & 0.278 & & \\
\hline THBSO: yes versus no & $1.86(0.69-5.00)$ & 0.222 & & \\
\hline Cholecystectomy: yes versus no & $0.46(0.11-1.96)$ & 0.294 & & \\
\hline Bladder resection: yes versus no & UD & 0.989 & & \\
\hline Other CRS procedure(s): yes versus no & $1.17(0.50-2.76)$ & 0.721 & & \\
\hline HIPEC agent: mitomycin $\mathrm{C}$ versus cisplatin & $0.48(0.20-1.13)$ & 0.093 & & \\
\hline HIPEC agent: others versus cisplatin & $1.67(0.37-7.62)$ & 0.508 & & \\
\hline Duration of CRS-HIPEC (per 10-min increase) & $1.01(0.98-1.04)$ & 0.469 & & \\
\hline CC score: $\geq 1$ versus 0 & UD & 0.990 & & \\
\hline Chest tube placement: yes versus no & $1.81(0.76-4.32)$ & 0.181 & & \\
\hline Stoma creation: yes versus no & $2.41(1.06-5.46)$ & 0.035 & $2.83(1.23-6.50)$ & 0.015 \\
\hline Blood loss (per 100-ml increase) & $0.99(0.95-1.04)$ & 0.730 & & \\
\hline Intraoperative blood transfusion: yes versus no & $1.16(0.47-2.85)$ & 0.743 & & \\
\hline Postoperative complication: G1-G2 versus none & $1.38(0.54-3.49)$ & 0.500 & & \\
\hline Postoperative complication: G3-G4 versus none & $2.42(0.79-7.39)$ & 0.122 & & \\
\hline Length of SICU stay (per day increase) & $0.96(0.79-1.15)$ & 0.631 & & \\
\hline Length of hospital stay (per day increase) & $1.01(0.99-1.03)$ & 0.194 & & \\
\hline
\end{tabular}

$H R$ hazard ratio, $C I$ confidence interval, CRS cytoreduction surgery, HIPEC hyperthermic intraperitoneal chemotherapy, ECOG Eastern Cooperative Oncology Group, $C O P D$ chronic obstructive pulmonary disease, $P C I$ peritoneal cancer index, THBSO total abdominal hysterectomy with bilateral salpingo-oophorectomy, $C C$ completeness of cytoreduction, SICU surgical intensive care unit, $U D$ no event in one of the categories

$p$ value based on Wald's test

${ }^{\mathrm{a}}$ Violated PH assumption 
FIG. 1. Kaplan-Meier curves of a DFS and $\mathbf{b}$ OS stratified by ERA, LRA, and NRA
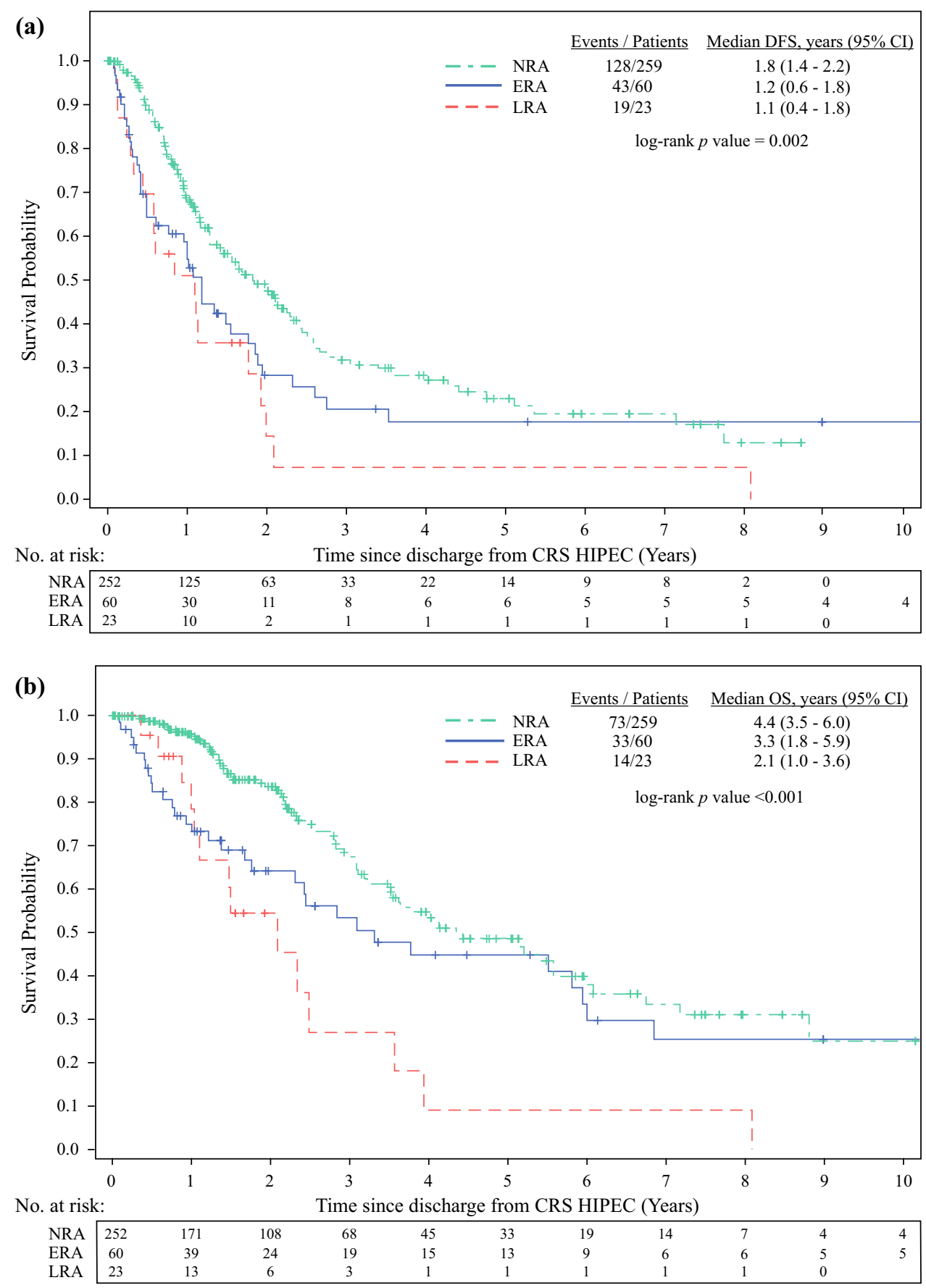

\section{DISCUSSION}

Internationally, reported rates of postoperative readmission after CRS-HIPEC range from 14.8 to $15.9 \%$ for ERA and from 3.9 to $11 \%$ for LRA. ${ }^{11,15,20}$ Known predictors include older age, number of previous surgical procedures, postoperative complications, and length of index hospitalization. ${ }^{10}$ Lee et al. went on to the compare the differences in predictors for RA at 30 versus 31-90 days. ECOG of 3 or more, intraoperative splenectomy, low anterior resection, partial colectomy, and stoma creation were independent predictors of 30-day RA, while gastric tumor, operative time, intraoperative low anterior resection or partial colectomy, and stoma creation were predictors for 31-90-day RA. ${ }^{11}$ Beyond 90 days, age and intraoperative colonic resection have been reported as the only independent risk factors for 6-month readmission. ${ }^{21}$ In our study cohort, we found similar rates of early and late readmission of $18.5 \%$ and $7.4 \%$, respectively, with a majority of RA occurring within 2 weeks post discharge. 
TABLE 6. Survival outcomes

\begin{tabular}{|c|c|c|c|c|c|}
\hline & Total $(N=342)$ & NRA $(n=259)$ & ERA $(n=60)$ & LRA $(n=23)$ & $p$ value* \\
\hline \multicolumn{6}{|l|}{ Follow-up duration (years) } \\
\hline Median (95\% CI) & $2.1(1.7-2.3)$ & $2.0(1.5-2.2)$ & $4.5(1.8-9.0)$ & 1.9 (0.8-NE) & 0.003 \\
\hline \multicolumn{6}{|l|}{ Disease-free survival (DFS) } \\
\hline No. of recurrences/deaths & 190 & 128 & 43 & 19 & 0.002 \\
\hline Median DFS, years $(95 \% \mathrm{CI})$ & $1.6(1.3-1.9)$ & $1.8(1.4-2.2)$ & $1.2(0.6-1.8)$ & $1.1(0.4-1.8)$ & \\
\hline 6-Month DFS, \% (95\% CI) & $82.7(78.0-86.5)$ & $88.8(83.9-92.3)$ & $64.0(50.3-74.8)$ & $69.6(46.6-84.2)$ & \\
\hline 1-Year DFS, \% (95\% CI) & $65.5(59.7-70.7)$ & $68.7(61.9-74.6)$ & $58.4(44.6-69.9)$ & $50.6(28.6-69.0)$ & \\
\hline 2-Year DFS, \% (95\% CI) & $41.4(35.1-47.7)$ & $48.1(40.4-55.3)$ & $28.0(16.2-41.1)$ & $14.2(2.6-35.1)$ & \\
\hline \multicolumn{6}{|l|}{ Overall survival $(O S)$} \\
\hline No. of deaths & 120 & 73 & 33 & 14 & $<0.001$ \\
\hline Median OS, years $(95 \% \mathrm{CI})$ & $3.9(3.2-5.4)$ & $4.4(3.5-6.0)$ & $3.3(1.8-5.9)$ & $2.1(1.0-3.6)$ & \\
\hline 1-Year OS, \% (95\% CI) & $90.7(86.7-93.5)$ & $95.8(92.0-97.8)$ & $75.1(61.6-84.5)$ & $78.6(52.0-91.5)$ & \\
\hline 2-Year OS, \% (95\% CI) & $77.8(71.9-82.6)$ & $83.6(77.0-88.5)$ & $64.1(49.4-75.6)$ & $54.4(28.8-74.2)$ & \\
\hline
\end{tabular}

$N E$ not estimable

*Based on log-rank test

Numbers in bold based on small no. of patients at risk

Only high-grade postoperative complications, stoma creation, and hypertension predicted ERA and LRA. In our study population, 57 patients $(16.7 \%)$ had high-grade complications, and 21 of these patients had ERA. In contrast, 176 patients $(51.5 \%)$ had risk factors for LRA, including those with both hypertension and stoma creation, hypertension only, and stoma creation only. Compared with patients with no LRA risk factors, risk of LRA was highest amongst patients with both hypertension and stoma creation $(\mathrm{HR}=7.67, n=23[6.7 \%])$, followed by patients with stoma creation only $[\mathrm{HR}=2.83, n=81(23.7 \%)]$ and patients with hypertension only [HR $=2.71, n=72$ $(21.1 \%)$ ], with our Cox model suggesting that these two risk factors have a multiplicative effect in predisposing patients to late readmission.

Postoperative readmission has been reported to be related to adverse survival outcomes in patients who had undergone surgery for cancers of various organs, such as brain, pancreas, esophagus, and stomach. ${ }^{22-25}$ Proposed contributors to this correlation include postoperative complications, infection, and metastatic disease. ${ }^{24,25}$ Others have also reported that readmission within 30 days of surgery is associated with delay in postoperative chemotherapy, which in turn is associated with poorer DFS and OS. ${ }^{26}$ In the context of post-pancreatic-cancer surgery, Reddy et al. reported that, compared with NRA, 0-30-day readmissions had lower median OS but comparable 5-year survival. Meanwhile 30-365-day readmissions had both lower median as well as 5-year survival compared with those without 30-365-day readmission. ${ }^{23}$ These findings suggested that, if the patients who required ERA survive the first few years following the index operation, their longterm outcome is comparable to those who had no readmission, whereas patients who required LRA have worse long-term outcomes regardless. We found that ERA and LRA patients had comparable median DFS, though significantly lower than NRA. Overall survival was worst amongst LRA, followed by ERA and NRA.

To the best of the authors' knowledge, this study is the first to compare survival outcomes in ERA, LRA, and NRA patients post CRS-HIPEC. Unfortunately, for our study, the median follow-up duration was not long enough to comment on 5-year survival outcomes. However, the survival curves at the 5-year mark seem to resonate the survival patterns reported by Reddy et al. The exact reason for these patterns of survival outcomes lies beyond the scope of this study. However, in broad conceptual terms, it may be reasonable to speculate that, post CRS-HIPEC, ERA is associated with potentially significant yet reversible causes, while LRA involves both significant and irreversible pathologies.

The adverse effect of postoperative morbidity on survival outcomes has been reported in literature for both oncological and nononcological surgeries. ${ }^{27-29}$ In the past, our center has also reported the association of high-grade complications with poor OS among post CRS-HIPEC patients; the 5-year OS rate of patients who experienced no postoperative, low-grade, and high-grade complications was found to be $52.8 \%, 37.0 \%$, and $43.0 \%$, respectively. ${ }^{30}$ As the results of the current study also show that high- 
grade morbidity is the sole independent risk factor for early readmission, it would be reasonable to infer that postoperative morbidity contributes to the association of ERA with poor survival outcomes.

Up to $30 \%$ of our CRS-HIPEC patients required stoma creation, a majority of which were defunctioning ileostomies for colorectal resection. Furthermore, intraoperative stoma creation was found to be a significant predictor of LRA, which was in turn associated with poorer survival outcomes. In patients with primary colorectal malignancies without peritoneal disease, post-stoma readmissions are known to be common and often occur within 30 days of discharge. ${ }^{31-33}$ Common early complications after stoma creation include skin irritation, pain, stoma retraction, and necrosis, while later in the clinical course, stoma patients may experience parastomal hernia, prolapse, stenosis, high output, and nutritional deficiencies. ${ }^{34,35}$ Our analysis of stoma formation in the setting of CRS-HIPEC found there was a greater percentage of stoma patients among the LRA than ERA group, from which we can cautiously infer that, for post CRS-HIPEC readmissions, stoma creation and its late complications may play a greater role in predisposing a patient to LRA. A small subgroup analysis of the 12 patients who were readmitted for stoma-related reasons showed that the vast majority of ERA was due to high stoma output $(83 \%)$, while for LRA only $50 \%$ presented with high stoma output and complications related to stoma reversal accounted for a sizable proportion (33\%). However, these figures are limited by small sample size, and a detailed analysis of stoma type, timing of reversal, related complications, and their association with readmissions and survival requires further investigation.

Based on the association of unplanned readmission with adverse survival outcomes seen in our results, future studies should investigate interventions that may reduce readmission and hence improve survival. One such intervention aimed to reduce LRA may include vigilant patient education and follow-up for those who have stoma creation during CRS-HIPEC, with special attention to known late complications such as parastomal herniations or post reversal strictures, infections, or anastomotic leak/breakdown. As our study also found that nearly half of all readmissions occurred within the first 15 days post discharge, with the majority presenting with gastrointestinal symptoms, it would be prudent to investigate whether early postoperative intervention with comprehensive discharge planning that includes appropriate discharge advice, streamlined wound care, dietician review, and close follow-up within the first month may help to off-load the high rates of ERA and provide survival benefits.

The retrospective design and relatively small number in this study may have resulted in selection bias and failure to elucidate other factors that may contribute to the differences in survival outcomes seen in the respective readmission groups. Longer follow-up may be required to further identify factors affecting long-term survival outcomes in our study population.

\section{CONCLUSIONS}

In this study conducted at the largest CRS-HIPEC center in Southeast Asia, unplanned postoperative readmission occurred at a rate of $18.5 \%$ for 30 days post discharge and $7.4 \%$ for 31-90 days. Unique sets of independent predictors were identified for these two readmission types: highgrade postoperative complication was a predictor of ERA, while stoma creation and hypertension were predictors for LRA. In addition, there were worse survival outcomes for patients with LRA as compared with ERA and NRA. Future studies may need to explore the association of poor survival with readmissions, to better identify effective measures to minimize unplanned hospitalizations post CRS-HIPEC.

ACKNOWLEDGMENT This study is funded by NCCS Cancer Fund. C.-A.J.O. is supported by the National Research Council Transition Award (NMRC/TA/0061/2017).

\section{DISCLOSURES None.}

OPEN ACCESS This article is licensed under a Creative Commons Attribution 4.0 International License, which permits use, sharing, adaptation, distribution and reproduction in any medium or format, as long as you give appropriate credit to the original author(s) and the source, provide a link to the Creative Commons licence, and indicate if changes were made. The images or other third party material in this article are included in the article's Creative Commons licence, unless indicated otherwise in a credit line to the material. If material is not included in the article's Creative Commons licence and your intended use is not permitted by statutory regulation or exceeds the permitted use, you will need to obtain permission directly from the copyright holder. To view a copy of this licence, visit http://creativecommons. org/licenses/by/4.0/.

\section{REFERENCES}

1. Huang C-Q, Min Y, Wang S-Y, et al. Cytoreductive surgery plus hyperthermic intraperitoneal chemotherapy improves survival for peritoneal carcinomatosis from colorectal cancer: a systematic review and meta-analysis of current evidence. Oncotarget. 2017;8(33):55657-83.

2. Chua TC, Moran BJ, Sugarbaker PH, et al. Early- and long-term outcome data of patients with pseudomyxoma peritonei from appendiceal origin treated by a strategy of cytoreductive surgery and hyperthermic intraperitoneal chemotherapy. J Clin Oncol. 2012;30(20):2449-56.

3. Huo YR, Richards A, Liauw W, Morris DL. Hyperthermic intraperitoneal chemotherapy (HIPEC) and cytoreductive surgery (CRS) in ovarian cancer: a systematic review and meta-analysis. Eur J Surg Oncol. 2015;41(12):1578-89. 
4. Sugarbaker PH, Jablonski KA. Prognostic features of 51 colorectal and 130 appendiceal cancer patients with peritoneal carcinomatosis treated by cytoreductive surgery and intraperitoneal chemotherapy. Ann Surg. 1995;221(2):124-32.

5. Wagner PL, Austin F, Maduekwe U, et al. Extensive cytoreductive surgery for appendiceal carcinomatosis: morbidity, mortality, and survival. Ann Surg Oncol. 2013;20(4):1056-62.

6. Bartlett EK, Meise C, Roses RE, Fraker DL, Kelz RR, Karakousis GC. Morbidity and mortality of cytoreduction with intraperitoneal chemotherapy: outcomes from the ACS NSQIP database. Ann Surg Oncol. 2014;21(5):1494-500.

7. Chua TC, Yan TD, Saxena A, Morris DL. Should the treatment of peritoneal carcinomatosis by cytoreductive surgery and hyperthermic intraperitoneal chemotherapy still be regarded as a highly morbid procedure? A systematic review of morbidity and mortality. Ann Surg. 2009;249(6):900-7.

8. Greenblatt DY, Weber SM, O'Connor ES, LoConte NK, Liou JI, Smith MA. Readmission after colectomy for cancer predicts oneyear mortality. Ann Surg. 2010;251(4):659-69.

9. Stitzenberg KB, Chang Y, Smith AB, Nielsen ME. Exploring the burden of inpatient readmissions after major cancer surgery. $J$ Clin Oncol. 2015;33(5):455-64.

10. Paredes AZ, Abdel-Misih S, Schmidt C, Dillhoff ME, Pawlik TM, Cloyd JM. Predictors of readmission after cytoreductive surgery and hyperthermic intraperitoneal chemotherapy. J Surg Res. 2019;234:103-9.

11. Lee TC, Wima K, Sussman JJ, et al. Readmissions after cytoreductive surgery and hyperthermic intraperitoneal chemotherapy: a US HIPEC collaborative study. $J$ Gastroint Surg. 2020;24(1):165-76.

12. Jafari MD, Halabi WJ, Stamos MJ, et al. Surgical outcomes of hyperthermic intraperitoneal chemotherapy: analysis of the American College of Surgeons National Surgical Quality Improvement Program. JAMA Surg. 2014;149(2):170-5.

13. Votanopoulos KI, Swords DS, Swett KR, et al. Obesity and peritoneal surface disease: outcomes after cytoreductive surgery with hyperthermic intraperitoneal chemotherapy for appendiceal and colon primary tumors. Ann Surg Oncol. 2013;20(12):3899-904.

14. Spiliotis J, Argiriou EO, Vafias E, et al. Re-admissions for delayed complications after cytoreductive surgery and HIPEC. Acta Chir Belg. 2016;116(2):96-100.

15. Martin AS, Abbott DE, Hanseman D, et al. Factors associated with readmission after cytoreductive surgery and hyperthermic intraperitoneal chemotherapy for peritoneal carcinomatosis. Ann Surg Oncol. 2016;23(6):1941-7.

16. Teo MC, Tan GH, Tham CK, Lim C, Soo KC. Cytoreductive surgery and hyperthermic intraperitoneal chemotherapy in Asian patients: 100 consecutive patients in a single institution. Ann Surg Oncol. 2013;20(9):2968-74.

17. Sugarbaker PH. Peritonectomy procedures. Ann Surg. 1995;221(1):29-42.

18. Jacquet $\mathrm{P}$, Sugarbaker PH. Clinical research methodologies in diagnosis and staging of patients with peritoneal carcinomatosis. Cancer Treat Res. 1996;82:359-74.

19. Dindo D, Demartines N, Clavien PA. Classification of surgical complications: a new proposal with evaluation in a cohort of 6336 patients and results of a survey. Ann Surg. 2004;240(2):205-13.

20. Dreznik Y, Hoffman A, Hamburger T, et al. Hospital readmission rates and risk factors for readmission following cytoreductive surgery (CRS) and hyperthermic intraperitoneal chemotherapy (HIPEC) for peritoneal surface malignancies. Surgeon. 2018;16(5):278-82.

21. Dranichnikov P, Graf W, Cashin PH. Readmissions after cytoreductive surgery and hyperthermic intraperitoneal chemotherapy - a national population-based study. World J Surg Oncol. 2020;18(1):67.

22. Dickinson $H$, Carico $C$, Nuño $M$, et al. Unplanned readmissions and survival following brain tumor surgery. J Neurosurg. 2015;122(1):61-8.

23. Fernandez FG, Khullar O, Force SD, et al. Hospital readmission is associated with poor survival after esophagectomy for esophageal cancer. Ann Thoracic Surg. 2015;99(1):292-7.

24. Merchant SJ, Ituarte $\mathrm{PH}$, Choi A, et al. Hospital readmission following surgery for gastric cancer: frequency, timing, etiologies, and survival. J Gatrointest Surg. 2015;19(10):1769-81.

25. Reddy DM, Townsend CM Jr, Kuo YF, Freeman JL, Goodwin JS, Riall TS. Readmission after pancreatectomy for pancreatic cancer in Medicare patients. $J$ Gastrointest Surg. 2009;13(11): 1963-74 (discussion 1974-1965).

26. Tevis SE, Kohlnhofer BM, Stringfield S, et al. Postoperative complications in patients with rectal cancer are associated with delays in chemotherapy that lead to worse disease-free and overall survival. Dis Colon Rectum. 2013;56(12):1339-48.

27. Zhou S, Feng Q, Zhang J, et al. High-grade postoperative complications affect survival outcomes of patients with colorectal cancer peritoneal metastases treated with cytoreductive surgery and hyperthermic intraperitoneal chemotherapy. BMC Cancer. 2021;21(1):41.

28. Li J, Zhang Y, Hu DM, Gong TP, Xu R, Gao J. Impact of postoperative complications on long-term outcomes of patients following surgery for gastric cancer: a systematic review and meta-analysis of 64 follow-up studies. Asian $J$ Surg. 2020;43(7):719-29.

29. Khuri SF, Henderson WG, DePalma RG, Mosca C, Healey NA, Kumbhani DJ. Determinants of long-term survival after major surgery and the adverse effect of postoperative complications. Ann Surg. 2005;242(3):326-41 (discussion 341-323).

30. Tan JW, Tan GHC, Ng WY, et al. High-grade complication is associated with poor overall survival after cytoreductive surgery and hyperthermic intraperitoneal chemotherapy. Int J Clin Oncol. 2020;25(5):984-94.

31. Messaris E, Sehgal R, Deiling S, et al. Dehydration is the most common indication for readmission after diverting ileostomy creation. Dis Colon Rectum. 2012;55(2):175-80.

32. Fish DR, Mancuso CA, Garcia-Aguilar JE, et al. Readmission after ileostomy creation: retrospective review of a common and significant event. Ann Surg. 2017;265(2):379-87.

33. Seo YJ, Bailey K, Aguayo E, et al. Readmissions after ileostomy creation using a nationwide database. Am Surg. 2018;84(10):1661-4.

34. Shabbir J, Britton DC. Stoma complications: a literature overview. Colorectal Dis. 2010;12(10):958-64.

35. Park JJ, Del Pino A, Orsay CP, et al. Stoma complications: the Cook County Hospital experience. Dis Colon Rectum. 1999;42(12):1575-80.

Publisher's Note Springer Nature remains neutral with regard to jurisdictional claims in published maps and institutional affiliations. 